\title{
Efficacy Considerations for U.S. Food and Drug Administration Approval of Diagnostic Radiopharmaceuticals
}

\author{
Alexander Gorovets, Louis Marzella, Dwaine Rieves, and Lucie Yang \\ Division of Medical Imaging Products, Office of Drug Evaluation IV, Center for Drug Evaluation and Research, U.S. Food and Drug \\ Administration, Silver Spring, Maryland
}

The safety and efficacy expectations for U.S. Food and Drug Administration (FDA) approval of diagnostic radiopharmaceuticals (DRs) are described in laws that broadly apply to all prescription drugs and biologic products. These laws also outline efficacy expectations that are unique for DRs. The FDA regulations and guidance documents elaborate on DR efficacy expectations for clinical uses of the drugs, such as the delineation of anatomy, the characterization of a physiologic process, or the diagnosis of disease. As described in the FDA regulations, the approval of a DR necessitates that the imaging drug has the ability to provide clinically useful information. Here we cite approved DRs to illustrate how the imaging performance of the drugs was characterized in clinical studies and the clinical usefulness of the imaging information described in drug labels.

Key Words: FDA; medical imaging drugs; molecular imaging; radiopharmaceuticals

J Nucl Med 2013; 54:1479-1484

DOI: 10.2967/jnumed.112.117804

\section{A}

broad array of radioactive compounds has been identified for development as diagnostic radiopharmaceuticals (DRs) with potentially important roles in the clinical care of patients (1). Critical to the development of these drugs is a clear understanding of the clinical efficacy considerations that the U.S. Food and Drug Administration (FDA) uses to assess the information submitted in marketing applications. The 2004 FDA guidance documents for medical imaging drugs describe these expectations in detail $(2,3)$. Using this guidance, we summarize here the key efficacy considerations for DRs and illustrate how these considerations were addressed for new drug applications and biologic license applications approved since the guidance documents were published. For more detailed information, we encourage readers to examine these guidance documents at the FDA Web site (www.fda.gov).

Federal law outlines the basis for the approval of DRs as drugs or biologic products, including the process by which marketing applications are submitted to the FDA. Specifically, the Federal Food, Drug, and Cosmetic Act (FDCA) sets the standard for the approval of a product as the FDA's finding that the product is safe

\footnotetext{
Received Nov. 28, 2012; revision accepted Feb. 15, 2013.

For correspondence contact: Louis Marzella, U.S. Food and Drug Administration, 10903 New Hampshire Ave, Silver Spring, MD 20993.

E-mail: Libero.Marzella@fda.hhs.gov

Published online Jun. 7, 2013.

COPYRIGHT (C) 2013 by the Society of Nuclear Medicine and Molecular Imaging, Inc.
}

and effective for its labeled indications and conditions of use (4). The FDA regulates DRs on the basis of sections of the FDCA, the Public Health Service Act (5), and regulations broadly applicable to prescription drugs as well as those addressed specifically to DRs. The regulations specific to DRs elaborate on the efficacy considerations for these products and describe the various types of DR indications that may be described in labeling. Here we use "labeling" to refer to a product's prescribing information, although labeling may include carton and vial labels and sometimes other documents.

The generation of clinical data to support a marketing application for a DR shares many process similarities with therapeutic drug development, such as the initial exploration of a drug's safety and activity in clinical trials with relatively small samples followed by the exposure of progressively more subjects in doseranging trials and ultimately confirmatory clinical trials. However, the efficacy considerations for a DR may differ substantially from those used in therapeutic drug development.

\section{EFFICACY CONSIDERATIONS FOR ALL PRESCRIPTION DRUGS AND BIOLOGIC PRODUCTS, INCLUDING DRS}

To support FDA approval of a DR, a sponsor must submit a marketing application: a new drug application for a drug or a biologic license application for a biologic product. Marketing applications for DRs are expected to contain the same general types of information as therapeutic product applications. On the basis of a review of the clinical data within the application, the FDCA requires the FDA to verify that the applicant has supplied "substantial evidence" of the effectiveness of the product as described in the proposed labeling (Fig. 1). The efficacy expectations for biologic products are similar to those for drugs, although biologic products are licensed under the authority of the Public Health Service Act. We highlight 3 particularly important concepts involved in the generation of clinical data for inclusion in marketing applications for new prescription drugs or biologic products, including DRs. Specifically, we refer to substantial evidence from adequate and well-controlled investigations as related to the proposed labeling of the product.

Substantial evidence is an evidentiary expectation that generally relates to the need for the independent substantiation of experimental results $(6)$. Independent substantiation of a product's efficacy may derive from 2 or more adequate and well-controlled clinical investigations as well as, in some situations, from a single adequate and well-controlled clinical investigation in which the product's efficacy is supported by other confirmatory evidence, such as clinical data that verify the product's efficacy in another patient population or another phase of a disease. 


\section{For all drugs:}

...Substantial evidence means evidence consisting of adequate and wellcontrolled investigations on the basis of which it could be concluded that the drug will have the effect it purports to have under the conditions of use prescribed in the labeling...

...In certain situations, data from one adequate and well-controlled investigation and confirmatory evidence may provide substantial evidence to establish effectiveness..

\section{Additionally, for diagnostic radiopharmaceuticals:}

...The determination of the safety and effectiveness shall include consideration of the proposed use of the radiopharmaceutical in the practice of medicine, the pharmacological and toxicological activity, and the estimated absorbed radiation dose of the radiopharmaceutical...

FIGURE 1. Key points from Federal Food, Drug, and Cosmetic Act and amendments (points are paraphrased; for full text, see references 4 and 10).

The characteristics of an adequate and well-controlled investigation are detailed in the FDA regulations (7). These regulations describe appropriate clinical study design features for placebocontrolled studies as well as concurrent no-treatment or activetreatment controlled studies. The regulations also cite the special situation in which historically controlled studies may be regarded as adequate and well controlled. In addition to clinical study design and conduct features, the regulations state that a product tested in an adequate and well-controlled investigation must be standardized with respect to its identity, strength, quality, purity, and dosage form. Standardization of product quality is an especially important consideration for an adequate and well-controlled study of a DR because some radionuclides are so short-lived that they must be produced at individual clinical site production facilities with procedures that have been standardized among all of the facilities. This circumstance is relatively unique to radiopharmaceuticals, and specific methods, facilities, and controls are used for the manufacturing and testing of the final drug products to ensure the necessary quality for medical imaging. Quick and efficient production may prove to be a priority to ensure that a drug product has sufficient radioactivity for its imaging purpose.

The FDA guidance on the development of a product's proposed labeling notes that the key objective in developing the prescribing information is to provide information that is most useful to prescribers in treating their patients (8). The FDA regulations describe the format for the presentation of prescribing information, including the nature of the information to include in specific sections (9). Among the various sections of the prescribing information, the "indications" and "clinical studies" sections are particularly notable for their derivation from the product's clinical efficacy data.

The FDA regulations describe how the "indications" section of a drug's prescribing information must state that the product is indicated for the treatment, prevention, mitigation, cure, or diagnosis of a recognized disease or condition or of a manifestation of a recognized disease or condition or for the relief of symptoms associated with a recognized disease or condition (9). The regulations allow this section to also include a description of the limitations of the product's usefulness as well as any uncertainty about the anticipated clinical benefits. The "clinical studies" section summarizes the major investigations supporting the product's effectiveness; that is, it describes the most important clinical studies that help characterize the product's clinical usefulness, not all of the clinical studies performed during the investigation of the product. This section may also summarize the important limitations of the available clinical effectiveness information.

\section{EFFICACY CONSIDERATIONS UNIQUE TO DRs}

The U.S. Food and Drug Administration Modernization Act of 1997 (10) amended the FDCA to include, among other items, a section pertaining to the marketing approval of DRs (Fig. 1). The amendment stated that the FDA should consider, among other items, the proposed use of a radiopharmaceutical in the practice of medicine and that the approved indications may refer to manifestations of disease (such as biochemical, physiologic, anatomic, or pathologic processes) (10). Subsequently, the FDA regulations noted that the effectiveness of a DR is assessed by evaluating its ability to provide useful clinical information related to its proposed use. The preamble to the regulation announcement illustrated how the FDA would recognize the ability to provide useful clinical information as being indicative of a clinical benefit (11). To exemplify this interpretation, the preamble cited a DR that located and outlined a normal parathyroid gland as providing clinically useful information because the image could indirectly assist a physician in planning surgery to remove a mass near the thyroid gland.

Four typical categories of medical imaging product indications are summarized in the FDA guidance, along with the recognition that indications outside these categories are possible (Fig. 2) (3). The guidance further describes the nature of the clinical efficacy data appropriate to support each type of indication.

The guidance also cites 2 major considerations as being essential to establishing the effectiveness of medical imaging products, including DRs: the accuracy of the imaging test and the clinical usefulness of the imaging test. Here we use excerpts of key concepts from the guidance documents and regulations to illustrate how these concepts were addressed in the clinical development of DRs approved after publication of the guidance documents.

Demonstration of the accuracy of a DR refers to the performance characteristics of the imaging test (such as sensitivity, specificity, and reproducibility), typically in comparison with a truth standard or a reference imaging product. In the absence of a truth standard or a reference product for the imaging test, the FDA guidance recommends that clinical studies validate the accuracy of the imaging information in terms of its impact on clinical outcomes. The establishment of DR performance characteristics would involve studies of populations of patients in which the imaging test would actually be used in medical practice; specifically, such studies would test the DR in subjects with and without a disease to cover the relevant spectrum of disease presentation. Similarly, if the DR measured a physiologic process

The labeled indications for medical imaging agents fall within the following general categories:

- Structure delineation

- Disease or pathology detection or assessment

- Functional, physiological, or biochemical assessment

- Diagnostic or therapeutic patient management.

Approval also may be possible for categories of indications not listed above.

FIGURE 2. Excerpts from FDA guidance on potential indications for medical imaging drugs. 
or a tissue component, then clinical studies would examine images from a population of subjects with a full distribution of potential image outcomes, including subjects with conditions that could affect image interpretation, such as inflammation or concomitant drug effects. The FDA guidance further describes how certain imaging indications, such as biochemical assessments, necessitate the identification of normal and abnormal ranges of outcome measures.

The clinical usefulness of the imaging information obtained with a DR, as reflected in the indication statement of the DR's prescribing information, necessitates identification of the clinical setting appropriate for performance of the imaging test. The FDA has recognized that the usefulness of imaging information may vary among clinical settings. Indication statements are expected to reflect the circumstances and conditions under which the imaging information is useful. This point is emphasized by the guidance recognition that, if the clinical implications of the imaging information are not understood, then generating an image will not provide clinically useful information and the role of the image in a clinical trial will be tenuous.

The FDA imaging product guidance recognizes how the clinical usefulness of some imaging information may be obvious in certain clinical settings, such as the staging of cancer or the detection of clinically important pathology. Additionally, the guidance notes that an indication related to the structural delineation of abnormal from normal anatomy can typically "speak for itself" with respect to the clinical usefulness of the information. For example, anatomic information is recognized as being useful in assisting surgeons during a dissection process. Furthermore, the guidance recognizes that, in some situations, a test that provides accurate information in describing a clinical condition is of well-established value. Specifically cited is the situation in which an imaging test is used for the detection of disease or pathology. In such situations, imaging drug developers are not expected to perform clinical studies that demonstrate again the clinical benefit of the imaging information.

Although DR developers do not need to demonstrate again the clinical benefits of imaging products in the defined clinical settings in which their usefulness can speak for itself, they are expected to demonstrate that the benefits of the imaging products justify their risks. With regard to assessing benefits and risks, the FDA guidance notes that risks include not only observed adverse reactions to a study drug but also inaccurate or misleading imaging information. For example, an inaccurate or misleading test may result in patients undergoing unnecessary or harmful additional tests. Ultimately, the major clinical considerations for the approval of a DR are similar to those for the approval of a therapeutic product in that clinical data must substantiate a favorable risk-tobenefit finding for the product in the context of the proposed labeling.

\section{DR NEW DRUG APPLICATIONS APPROVED SINCE 2004}

The FDA has approved 9 marketing applications for new DRs since the medical imaging guidance documents were published in 2004 (Table 1, cumulative through September 15, 2012). Three of these products, Ammonia N 13 Injection and Fludeoxyglucose F 18 Injection (2 separate applications), were approved with citation to a prior FDA finding of efficacy for certain uses of the drugs, as published in the Federal Register (12). The approval of Sodium Fluoride F 18 Injection was based on the FDA approval of another sodium fluoride product in 1972. The one licensed biologic product, Kit for the Preparation of Technetium (99m Tc) Fanolesomab, was withdrawn from marketing by the manufacturer after reports of serious reactions to the product (13). For the remaining drugs, we summarize the key confirmatory clinical efficacy data and discuss how these data verified the accuracy and the clinical usefulness of the imaging information. Additional information is available in the "clinical studies" section of the prescribing information for each drug. Details of the FDA review of the clinical data for several of the drugs are also available at the FDA Web site (14).

\section{lobenguane I 123 Injection}

The 2007 approval of Iobenguane I 123 Injection included labeling that cited the drug's usefulness in the detection of neuroblastoma or pheochromocytoma. This indication is typical of the disease or pathology detection indication category described in the FDA guidance. The drug developer established the drug's efficacy through submission of the results of a single confirmatory clinical trial plus the results of a review of published study reports. The confirmatory trial used an open-label, multicenter approach to enroll patients with known or suspected neuroblastoma or pheochromocytoma. The accuracy (sensitivity and specificity) of imaging with the drug was assessed by comparison of images to a truth standard that consisted of histopathology plus other cancer markers, such as plasma or urine catecholamine levels as well as clinical follow-up of the patients. The trial established acceptable accuracy of the DR, as denoted by the description in the prescribing information of the sensitivity and specificity of imaging with the DR. The clinical usefulness of the imaging information (cancer localization) was accepted by the FDA as self-evident, consistent with the disease detection perspective outlined in the FDA guidance.

\section{loflupane I 123 Injection}

The Ioflupane I 123 Injection clinical development program targeted the use of the drug to imaging of the brain dopamine transporter (DAT) distribution to help in the diagnostic evaluation of patients with tremors. The program illustrates 2 considerations that may sometimes challenge the development of a molecular imaging agent: the logistic difficulties that may preclude the use of histopathology as a truth standard and the need to evaluate patients over a period of time to establish the clinical usefulness of the molecular imaging agent. To address these challenges, the drug's developer integrated in vivo and in vitro ${ }^{123} \mathrm{I}$-ioflupane-DAT binding information with nonclinical and clinical neurophysiologic data to support the contentions that the brain DAT images could be categorized as showing either normal or abnormal findings and that this information could be used to assist in the diagnostic evaluation of patients with tremors. According to this proposal, the imaging information would assist clinicians in attributing the tremors to either a parkinsonian syndrome (PS) or a non-PS condition. Abnormal findings on a brain DAT image were proposed as being typical for the PS condition, in contrast to a typically normal image for a patient with a non-PS condition.

The Ioflupane I 123 clinical trial reference standard was a dichotomous classification of a patient with tremors as having either a PS or a non-PS condition, on the basis of clinical followup evaluations; this clinical classification was accepted as an alternative to a histopathology truth standard because background nonclinical and clinical data had shown that patients with a PS have abnormally low brain striatal DAT receptor levels in comparison 
TABLE 1

DRs Approved (New Drug Application) or Licensed (Biologic License Application) by FDA Since Publication of Medical Imaging Guidance Documents

\begin{tabular}{|c|c|c|}
\hline $\begin{array}{l}\text { Year(s) of } \\
\text { approval } \\
\text { or licensure }\end{array}$ & Product & Indication and key portions of text excerpted from prescribing information \\
\hline 2004 & $\begin{array}{l}\text { Kit for the Preparation of } \\
\text { Technetium ( } 99 \mathrm{~m} \text { Tc) } \\
\text { Fanolesomab }\end{array}$ & Scintigraphic imaging of patients with equivocal signs and symptoms of appendicitis \\
\hline $\begin{array}{l}2004 \text { and } \\
2005^{\star}\end{array}$ & $\begin{array}{l}\text { Fludeoxyglucose } \\
\text { F } 18 \text { Injection }\end{array}$ & $\begin{array}{l}\text { Assessment of abnormal glucose metabolism to assist in evaluation of malignancy in } \\
\text { patients with known or suspected abnormalities found by other testing modalities } \\
\text { or in patients with existing diagnosis of cancer } \\
\text { Identification of left ventricular myocardium with residual glucose metabolism and } \\
\text { reversible loss of systolic function in patients with coronary artery disease and } \\
\text { left ventricular dysfunction, when used together with myocardial perfusion imaging } \\
\text { Identification of regions of abnormal glucose metabolism associated with foci of } \\
\text { epileptic seizures }\end{array}$ \\
\hline 2007 & $\begin{array}{l}\text { Ammonia N } 13 \\
\text { Injection }\end{array}$ & $\begin{array}{l}\text { Diagnostic PET imaging of myocardium under rest or pharmacologic stress conditions to } \\
\text { evaluate myocardial perfusion in patients with suspected or existing coronary artery disease }\end{array}$ \\
\hline 2008 & $\begin{array}{l}\text { lobenguane I } 123 \\
\text { Injection }\end{array}$ & $\begin{array}{l}\text { Detection of primary or metastatic pheochromocytoma or neuroblastoma as adjunct } \\
\text { to other diagnostic tests }\end{array}$ \\
\hline 2011 & $\begin{array}{l}\text { loflupane I } 123 \\
\text { Injection }\end{array}$ & $\begin{array}{l}\text { Striatal DAT visualization with SPECT brain imaging to assist in evaluation of adult } \\
\text { patients with suspected PS; in these patients, DaTscan may be used to help } \\
\text { differentiate essential tremors from tremors attributable to PS }\end{array}$ \\
\hline 2011 & $\begin{array}{l}\text { Sodium Fluoride F } 18 \\
\text { Injection }\end{array}$ & PET imaging of bone to define areas of altered osteogenic activity \\
\hline 2012 & $\begin{array}{l}\text { Choline C } 11 \\
\text { Injection }\end{array}$ & $\begin{array}{l}\text { PET imaging of patients with suspected prostate cancer recurrence and noninformative } \\
\text { bone scintigraphy, CT, or MR imaging findings; in these patients, }{ }^{11} \mathrm{C} \text {-choline PET } \\
\text { imaging may help identify potential sites of prostate cancer recurrence for } \\
\text { subsequent histologic confirmation; suspected prostate cancer recurrence is based } \\
\text { on elevated blood prostate-specific antigen levels after initial therapy } \\
\text { Limitations of use: }{ }^{11} \mathrm{C} \text {-choline PET imaging does not replace histologic verification of } \\
\text { recurrent prostate cancer }\end{array}$ \\
\hline 2012 & $\begin{array}{l}\text { Florbetapir F } 18 \\
\text { Injection }\end{array}$ & $\begin{array}{l}\text { PET imaging of brain to estimate } \beta \text {-amyloid neuritic plaque density in adult patients } \\
\text { who have cognitive impairment and are being evaluated for AD and other causes of } \\
\text { cognitive decline; negative Amyvid scan findings indicate sparse to no neuritic } \\
\text { plaques and are inconsistent with neuropathologic diagnosis of AD at time of image } \\
\text { acquisition; negative scan findings reduce likelihood that patient's cognitive } \\
\text { impairment is attributable to AD; positive Amyvid scan findings indicate moderate } \\
\text { to frequent amyloid neuritic plaques; neuropathologic examination has shown that } \\
\text { such quantities of amyloid neuritic plaques are present in patients with AD but may } \\
\text { also be present in patients with other types of neurologic conditions as well as } \\
\text { older people with normal cognition } \\
\text { Limitations of use: positive Amyvid scan findings do not establish diagnosis of AD or } \\
\text { other cognitive disorder; safety and effectiveness of Amyvid for predicting } \\
\text { development of dementia or other neurologic conditions or for monitoring } \\
\text { responses to therapies have not been established }\end{array}$ \\
\hline
\end{tabular}

*One new drug application was approved in 2004, and another was approved in 2005; excerpt is from updated labeling for drug approved in 2005. Not included are drugs approved under abbreviated new drug applications (i.e., "generic" drugs). Reference 14 provides full prescribing information for each drug.

with patients with a non-PS condition. This reference standard also helped verify the clinical usefulness of the imaging information because earlier (rather than later) determination of whether a patient's tremors are attributable to a PS or a non-PS condition has well accepted therapeutic and prognostic implications.

In one of the confirmatory Ioflupane I 123 clinical studies, the diagnostic evaluations were performed over a 3-y period after imaging; this time period was sufficient to allow movement disorder specialists to definitively assign to each patient a PS or a non-PS diagnosis. The baseline Ioflupane I 123 images were subsequently compared with this diagnostic reference standard (PS or non-PS). On the basis of the clinical trial findings, the Ioflupane I 123 Injection prescribing information describes the accuracy of imaging with Ioflupane I 123 in terms of agreement between the imaging results (normal or abnormal) and the reference standard (PS or non-PS diagnosis). The drug was approved in 2011, and its clinical usefulness is further described in the indication statement of the prescribing information by a notation that the visualization of 
brain striatal DAT may assist in the evaluation of adult patients with a suspected PS by helping to differentiate essential tremors from tremors attributable to a PS. Hence, the clinical usefulness of imaging with Ioflupane I 123 was confined to the setting of uncertainty as to whether a patient with tremors had a PS or not.

\section{Florbetapir F 18 Injection}

The 2012 approval of Florbetapir F 18 Injection for brain amyloid imaging in certain settings followed a development program that involved extensive public discussion of the assessment of the drug's accuracy and potential clinical usefulness. On the basis of in vitro and in vivo data indicating that certain molecular probes could bind to brain amyloid, drug developers approached the FDA in 2007 to assist in the development of amyloid imaging agents. The challenges encountered with these agents were somewhat similar to those encountered with Ioflupane I 123, in that a brain histopathology truth standard raised feasibility questions and the clinical usefulness of brain amyloid imaging information was unclear. These topics were the focus of a 2008 public meeting of the FDA Peripheral and Central Nervous System Advisory Committee. This committee advised the FDA that a brain amyloid imaging drug could have clinical usefulness in helping to rule out a diagnosis of Alzheimer disease (AD) when imaging results are read as negative and that histopathology should be used as the truth standard for phase 3 studies despite some concern about the feasibility of obtaining brain tissue.

The developer of Florbetapir F 18 Injection established the drug's efficacy with 3 confirmatory studies. One study correlated amyloid levels on premortem images, as scored on a 5-point scale, with a postmortem semiquantitative measure of whole-brain amyloid content. That study, which used a research-based image interpretation method, helped verify that the DR actually imaged brain amyloid. Two other confirmatory studies used a clinically applicable image reading method in which images were assessed as showing either positive or negative results on the basis of specific patterns of radioisotope signals. The negative or positive findings on images were compared with a truth standard consisting of the postmortem histopathologic determination of whether a patient's brain contained a neuropathologically important brain amyloid plaque density (a dichotomous outcome). These 2 clinical studies differed from each other in the reader training method (in-person vs. electronic self-study), but both determined accuracy (sensitivity and specificity) relative to whole-brain histopathology at autopsy. One of the studies also enrolled subjects without a truth standard, including healthy adults as well as subjects with mild cognitive impairment or clinically diagnosed AD. In both studies, image interpretation agreement among and within readers (reproducibility) was an important consideration because the proposed image interpretation methods differed from commonly used nuclear medicine image interpretation methods. Together, the studies confirmed that the DR imaged brain amyloid plaque in a manner conducive to clinical use, with acceptable reproducibility of image interpretation (with either in-person or electronic self-study training) as well as acceptable accuracy.

In addition to presenting data on the accuracy and reproducibility of amyloid imaging, the prescribing information for Florbetapir F 18 Injection describes how the estimation of brain amyloid plaque density on images is clinically useful. Specifically, negative findings on a scan are inconsistent with a neuropathologic diagnosis of $\mathrm{AD}$ at the time of imaging; such findings reduce the likelihood that a patient's cognitive impairment is attributable to AD. Hence, negative findings on a scan may enhance efforts to detect a non-AD cause of a patient's cognitive decline, such as severe depression. This outcome (negative findings on a scan) in a clinically uncertain situation forms the basis for the clinical usefulness of the scan.

Because brain amyloid may accumulate in people who are cognitively normal and because the correlation between amyloid content and clinical disease manifestations is unknown, the indication statement of the drug's prescribing information includes a description of important limitations of the imaging information. Specifically, the prescribing information notes that positive results on a scan do not diagnose any cognitive disorder and that studies have not established any role for the scan in predicting the development of neurologic conditions or in monitoring responses to therapies.

\section{Choline C 11 Injection}

Choline C 11 Injection, the most recently approved DR, is indicated for use in helping to identify potential sites of prostate cancer recurrence in patients with suspected recurrence and noninformative bone scintigraphy, CT, or MR imaging findings. As noted for Iobenguane I 123 Injection, the ability of the imaging information obtained with a DR to help localize a cancer site represents a disease detection claim-imaging information typically associated with implicit clinical usefulness, according to the FDA guidance. Hence, one of the main challenges in the Choline C 11 Injection development program was to obtain a reasonable estimate of the accuracy of the imaging information in the specific clinical setting applicable to its indication.

Relative to the 3 new drugs described here, Choline C 11 was unique in that it had been in clinical use-including use outside investigations typically conducted after the submission of investigational new drug applications to the FDA-for a few years before the submission of a marketing application. As a PET drug, Choline C 11 is subject to the components of the U.S. Food and Drug Administration Modernization Act of 1997 (10), which set a time line for the submission of marketing applications or investigational new drug applications to support the clinical use of unapproved PET drugs. The marketing application for Choline $\mathrm{C} 11$ Injection included a systematic review of published reports of studies that had used the DR, including detailed information from the applicant's clinical experience with the drug (15).

The efficacy of Choline C 11 Injection was based on published reports in which imaging results were compared with a histopathology truth standard (cancer or no cancer, after biopsy or resection of a suspected lesion). From a review of all published reports within a designated publication database, 4 publications were found to describe studies that compared imaging results with a histopathology truth standard in a clinical setting in which conventional imaging modalities had been noninformative (i.e., the other imaging tests had failed to find a potential site of cancer recurrence in patients whose serum prostate-specific antigen levels had suggested recurrence after primary therapy). In each of the 4 studies, at least half the patients for whom abnormalities were found on Choline C 11 PET scans also had recurrent prostate cancer confirmed by tissue sampling of the areas with abnormalities. The accuracy of the PET scans was described in the prescribing information in terms of the distribution of true or false scan results relative to the truth standard. These data showed that false-positive results on PET scans were observed in $15 \%-47 \%$ of the patients, depending on the study. The strengths and limitations of the performance of the scans necessitated the description in the prescribing information of an important limitation of the use of the scans: 
" ${ }^{11} \mathrm{C}$-choline PET imaging is not a replacement for histologic verification of recurrent prostate cancer" (16). This experience also underscores the importance of careful consideration of a DR's potential labeling as the drug development proceeds, especially for DRs that may have been in clinical use even though the specific usefulness of those DRs had not been characterized.

\section{CONCLUSION}

The recent history of DR approvals illustrates how the drug developers obtained clinical efficacy data that characterized not only the accuracy of the imaging information but also how that information is clinically useful. In the examples described here, establishing the accuracy of DR imaging outcomes was generally straightforward and was based on various methods of performance characteristic assessment, such as measures of sensitivity, specificity, reproducibility, and predictive value. However, the examples also illustrated that defining specifically how the imaging information obtained with a DR is clinically useful may prove to be more challenging. This consideration may be particularly applicable to DR ligands that have been widely recognized as characterizing pathophysiologic processes in disease-yet specifically how imaging with the ligands provides clinically useful information for a patient has not been determined. For FDA approval to be obtained, a DR clinical database needs more than a conceptual understanding of the importance of the DR ligand in human pathophysiology. As illustrated in the examples described here, the DR developer must consider several logistic concerns, such as identification of the clinical setting appropriate for use of the imaging test, how images should be interpreted, and specifically how imaging results provide clinically useful information.

Describing how an imaging test provides useful information may prove to be a key initial consideration for a developer who seeks to obtain FDA approval of a DR. Hence, the clinical development program for a DR may benefit from early consideration of the potential labeling of the DR. Although labeling may be one of the last steps in the preparation of a new drug application, it may prove to be especially useful to link drug development activities to specific aspects of the proposed drug labeling.

The 4 recent DR approvals described here demonstrate the potential for different categories of indications; for marketing application content that ranges from clinical studies conducted by the applicant to reports from the published literature; for the use of a clinical trial reference standard other than pathology; and for various ways of describing, in the labeling of the DR, how the DR may be clinically useful.

The FDA's approval of DRs is based on risk and benefit considerations broadly applicable to prescription drugs and biologic products as well as considerations unique to diagnostic imaging drugs. DR development also involves certain risk assessment aspects not addressed here, such as the need to establish the radiation safety, overall safety, and quality of production of the drug. The FDA Web site (www.fda.gov) contains many documents that address these items in considerable detail, and we encourage developers to examine these resources.

\section{DISCLOSURE}

The costs of publication of this article were defrayed in part by the payment of page charges. Therefore, and solely to indicate this fact, this article is hereby marked "advertisement" in accordance with 18 USC section 1734. The views expressed in this article are the result of independent work and do not necessarily represent the views and findings of the U.S. Food and Drug Administration. No potential conflict of interest relevant to this article was reported.

\section{REFERENCES}

1. Hall LT, Struck AF, Perlman SB. Clinical molecular imaging with PET agents other than ${ }^{18}$ F-FDG. Curr Pharm Biotechnol. 2010;11:545-554.

2. U.S. Food and Drug Administration. Guidance for industry: developing medical imaging drug and biological products, part 3: design, analysis, and interpretation of clinical studies. Available at: http://www.fda.gov/downloads/Drugs/ GuidanceComplianceRegulatoryInformation/Guidances/UCM071604.pdf. Accessed September 3, 2012.

3. U.S. Food and Drug Administration. Guidance for industry: developing medical imaging drug and biological products, part 2: clinical indications. Available at: http://www.fda.gov/downloads/Drugs/GuidanceComplianceRegulatoryInformation/ Guidances/ucm071603.pdf. Accessed September 3, 2012.

4. Federal Food, Drug and Cosmetic Act \$201(p)(1) and 505(d), 21 USC 321(p)(1) and 355(d) (2012)

5. Public Health Service Act $\$ 351,42$ USC 262 (2012).

6. U.S. Food and Drug Administration. Guidance for industry: providing clinical evidence of effectiveness for human drugs and biological products. Available at: http:/www.fda.gov/downloads/Drugs/GuidanceComplianceRegulatoryInformation/ Guidances/UCM078749.pdf. Accessed September 3, 2012

7. 21 Code of Federal Regulations, Part 314.126.

8. U.S. Food and Drug Administration. Guidance for industry: clinical studies section of labeling for human prescription drug and biological productscontent and format. Available at: http://www.fda.gov/downloads/Drugs/ GuidanceComplianceRegulatoryInformation/Guidances/ucm075059.pdf. Accessed September 3, 2012

9. 21 Code of Federal Regulations, Parts 201.56, 201.57, and 314.50.

10. Public Law 105-115. U.S. Food and Drug Administration Modernization Act of 1997. Available at: http://www.gpo.gov/fdsys/pkg/PLAW-105publ115/pdf/ PLAW-105publ115.pdf. Accessed May 23, 2013.

11. U.S. Food and Drug Administration. Regulations for in vivo radiopharmaceuticals used for diagnosis and monitoring: final rule. Fed Regist. 1999;64:26657-26670.

12. Products PETD. Safety and effectiveness of certain PET drugs for specific indications. Fed Regist. 2000;65:12999-13010.

13. NeutroSpec (technetium [99m Tc] fanolesomab) [FDA press release]. Available at: http://www.fda.gov/Safety/MedWatch/SafetyInformation/SafetyAlertsforHumanMedicalProducts/ucm152014.htm. Accessed September 25, 2012.

14. Drugs@FDA: FDA approved drug products. U.S. Food and Drug Administration Web site. Available at: http://www.accessdata.fda.gov/scripts/cder/drugsatfda/ index.cfm. Accessed May 10, 2013.

15. Positron emission tomography (PET). U.S. Food and Drug Administration Web site. Available at: http://www.fda.gov/Drugs/DevelopmentApprovalProcess/Manufacturing/ ucm085783.htm. Accessed May 10, 2013.

16. Drugs@FDA: FDA Approved Drug Products. Choline C-11, NDA 203155. U.S. Food and Drug Administration Web site. Available at: http://www.accessdata.fda. gov/scripts/cder/drugsatfda/index.cfm?fuseaction=Search.DrugDetails. Accessed May 20, 2013 